Artículo de Revisión

\title{
COMPLICACIONES RESPIRATORIAS PERIOPERATORIAS
}

\author{
Mauricio Ramos G. ${ }^{1}$
}

Key words: Pulmonary complications; intraoperative atelectasis; lung injury; ARDS; tidal volume; plateau pressure; inspiratory muscle training; pneumonia; respiratory failure.

\section{Clasificación}

La morbilidad respiratoria representa un capítulo muy trascendente de la medicina perioperatoria. Si bien no ha tenido un estudio tan sistematizado, su incidencia, la prolongación de la estadía hospitalaria que provoca y su mortalidad la hacen equipararse en importancia a la morbilidad cardiovascular.

Los estudios publicados tienen una variabilidad en las definiciones y en la metodología usadas, por lo que es útil enmarcarse en los eventos mayores que consideró Fisher ${ }^{1}$ en su estudio de 2002 que son la insuficiencia respiratoria que, por definición, requiere apoyo ventilatorio invasivo o no invasivo, la neumonía, los episodios de broncoespasmo, las atelectasias y derrames pleurales que requieren intervención terapéutica como la broncoscopia. No consideró la tos productiva y las alteraciones radiológicas, lo cual implica todas aquellas atelectasias menores que son clínicamente silentes. Por supuesto, a la luz de la realidad clínica actual, es necesario agregar a este grupo de eventos mayores el Distress Respiratorio del Adulto.

La mortalidad de los eventos mayores es muy alta, alcanzando un $21 \%$ para la neumonía y $27 \%$ para la insuficiencia respiratoria. Estas complicaciones prolongan la estadía intrahospitalaria en promedio dos semanas $1,2,3,4,5,6$.

Claramente la cirugía intratorácica (corazón, mediastino, pulmón) es un subgrupo muy importante en este contexto, pero representa un porcentaje menor de la práctica quirúrgica global. La suma de las complicaciones respiratorias alcanza a un $30 \%$ de los pacientes sometidos a cirugía no torá- cica $^{2,3,4,5,6}$. La incidencia de las diferentes complicaciones por separado se ha obtenido de una serie importante de publicaciones.

La insuficiencia respiratoria, con necesidad de apoyo ventilatorio invasivo o no invasivo, alcanza entre 2,3 y $3,4 \% \%^{5,7,8}$. La neumonía afecta entre el 1,5 y $3,3 \% 0^{6,7,8}$ de los pacientes. La incidencia de atelectasias con manifestación clínica (no sólo radiológica) y que, por definición, requieren intervención broncoscópica, es de $2,5 \%$ según el estudio de McAlister ${ }^{7}$ y de 1,4\% según el de Canet $^{8}$. Para el broncoespasmo se han publicado incidencias entre $0,6 \%{ }^{9}$ y $1,8 \%{ }^{8}$. El distress respiratorio post cirugía torácica es $2,5 \%$ para todo tipo de resección de parénquima pulmonar y $7,9 \%$ para las neumonectomías $^{10}$.

\section{Factores de riesgo}

Una serie de publicaciones ${ }^{2-9,11-14}$ han permitido configurar un cuadro bastante completo que permite identificar factores asociados al paciente, al tipo de cirugía y a la anestesia, como lo muestra Tunsman ${ }^{15}$ en su revisión de 2012.

En la Tabla 1 hay una numerosa lista de factores y condiciones del paciente que se asocian a un mayor riesgo respiratorio y, entre éstos, destacan ciertos antecedentes clínicos.

Uno de ellos es la limitación crónica al flujo aéreo ${ }^{16-20}$, cuyos pacientes tienen una incidencia de $23 \%$ de complicaciones y un riesgo relativo (RR) de $3,0^{9,21}$ dentro de lo que destaca una incidencia de broncoespasmo de hasta $37 \%$. Un factor importante es la fatiga muscular respiratoria de estos

Instituto Nacional del Tórax, Santiago.

Hospital de Urgencia Asistencia Pública.

Clínica Alemana. 
Tabla 1. Factores y condiciones asociadas a mayor riesgo de complicaciones respiratorias perioperatorias

\begin{tabular}{|c|c|c|}
\hline Factores del paciente & Tipo de cirugía & Factores anestésicos \\
\hline Edad: $>65$ años o $<6$ años & Vascular & Balance líquido positivo \\
\hline $\mathrm{ASA} \geq 3$ & Torácica & Transfusión $>4$ unidades \\
\hline LCFA & Abdomen superior & $\mathrm{Vt} \uparrow-$ presión meseta $\uparrow$ \\
\hline Tabaquismo & Neurocirugía & Anestesia /Analgesia Peridural \\
\hline $\mathrm{SpO}_{2} \%$ preoperatoria & Orofacial y cervical & Sonda nasogástrica \\
\hline Insuficiencia cardiaca congestiva & Urgencia & Soporte ventilatorio postoperatorio \\
\hline Infección respiratoria menos de 1 mes & Duración $>3$ horas & Relajante muscular de larga duración \\
\hline Dependencia funcional & Abierta/laparoscópica & Hipotermia \\
\hline Alteración función renal & & Anestesia intravenosa $v s$ inhalatoria \\
\hline Abuso de alcohol & & Mascarilla $v s$ tubo endotraqueal \\
\hline \multicolumn{3}{|l|}{ Hipertensión Pulmonar } \\
\hline \multicolumn{3}{|l|}{ Pérdida peso $>10 \%$} \\
\hline \multicolumn{3}{|l|}{$\mathrm{BMI}>27 \mathrm{~kg} \cdot \mathrm{m}^{2}$} \\
\hline \multicolumn{3}{|l|}{ Apnea obstructiva del sueño } \\
\hline \multicolumn{3}{|l|}{ Albúmina $<3,5 \mathrm{~g} \bullet \mathrm{dl}^{-1}$} \\
\hline \multicolumn{3}{|l|}{ Hemoglobina $<10 \mathrm{~g} \bullet \mathrm{dl}^{-1}$} \\
\hline \multicolumn{3}{|l|}{ Alteración del sensorio } \\
\hline Examen torácico anormal & & \\
\hline
\end{tabular}

pacientes, por lo que se hace importante el entrenamiento muscular, el control del estado nutritivo y de los desórdenes electrolíticos ${ }^{23}$.

El tabaquismo es otro de los factores de riesgo, debidos a sus efectos per se y por su asociación a enfermedad pulmonar crónica ${ }^{10,12,24-27}$. La suspensión de este hábito por 48 horas permite normalizar los niveles de carboxihemoglobina y de nicotina, así como recuperar el movimiento ciliar. Con 2 a 3 semanas de suspensión del cigarrillo comienzan a mejorar las secreciones bronquiales. Los síntomas y la función pulmonar comienzan a mejorar después de las 4-6 semanas y recién a las 8 semanas de suspensión comienza a disminuir el riesgo ${ }^{3}$.

La obesidad representa un tipo de enfermedad respiratoria restrictiva. En estos pacientes la capacidad residual funcional es menor que la capacidad de cierre, con una alta distensibilidad de la vía aérea superior, que favorece la producción de ronquido y apnea obstructiva del sueño, y que se asocian a apnea y desaturación postoperatorias ${ }^{7,28,29,30}$. La mortalidad por insuficiencia respiratoria es prácticamente el doble en personas obesas (30\% vs 17\%).

Las cirugías sobre estructuras intratorácicas tienen una incidencia de complicaciones respiratorias mucho más alta que la cirugía general, digestiva u ortopédica $^{8}$. La cirugía de abdomen alto y, en es- pecial, la cirugía vascular son también poblaciones de alto riesgo. Sin embargo, el tipo de cirugía es un elemento que no se puede modificar, a diferencia de los factores y condiciones clínicas que pueden ser controladas y/o mejoradas antes de la cirugía.

En lo que respecta a la técnica anestésica hay variables que se pueden considerar para mejorar la expectativa respiratoria o, al menos, para no empeorar la situación.

En términos generales, la anestesia general, permite el control de la vía aérea y de las secreciones pero, como contraparte, altera el control del centro respiratorio, hay manipulación de vía aérea y existe el riesgo de bloqueo muscular residual.

Varios estudios han evaluado la resistencia de la vía aérea después de la inducción de la anestesia. En términos de inductores, el propofol provoca un menor aumento de la resistencia, tanto en fumadores como no fumadores, y se ha reportado una menor incidencia de broncoespasmo ${ }^{31,32,33,34}$. La ketamina $^{35,36}$ es una buena alternativa cuando la hemodinamia está comprometida, ya que la resistencia de la vía aérea puede ser incluso menor que con el uso de propofol.

Una medida efectiva para disminuir la resistencia de la vía aérea es el uso combinado de lidocaína y salbutamol, que tienen un efecto sinérgico sobre 
la musculatura bronquial ${ }^{37}$.

La manipulación de la vía aérea es un punto importante. El uso de máscara laríngea evita que la resistencia aumente inicialmente como ocurre con la intubación endotraqueal ${ }^{38,39}$.

El efecto residual de los bloqueadores neuromusculares se ha asociado a eventos respiratorios ${ }^{12}$. En este aspecto los de duración prolongada tienen más probabilidad de producir bloqueo residual. Además, algunos bloqueadores neuromusculares pueden liberar histamina y estar relacionados con la producción de broncoespasmo. Existe un índice que es la dosis de relajante que duplica la concentración plasmática de histamina en el $50 \%$ de los pacientes $\left(\mathrm{DA}_{50}\right)$. En los fármacos pertenecientes a la familia de las benzilisoquinolinas, atracurio ${ }^{40,41} \mathrm{y}$ mivacurio ${ }^{42}$ la $\mathrm{DA}_{50}$ corresponde entre 1 y 3 veces la dosis $\mathrm{ED}_{95}$. El margen de seguridad con doxacurio y cisatracurio es mayor, ya que se requieren 4 y 8 veces su dosis $\mathrm{ED}_{95}$ para el mismo efecto.

Los agentes inhalatorios halogenados tienen una característica muy positiva en este contexto, cual es su efecto broncodilatador. Si bien diversos trabajos comparativos no muestran diferencias importantes entre isofluorano, sevofluorano y desfluorano $\mathrm{o}^{43,44,45}$, existen algunos antecedentes que muestran cierto mayor efecto de isofluorano comparado con sevofluorano y de éste comparado con desfluorano ${ }^{46,47}$.

$\mathrm{La}$ anestesia regional tiene la ventaja de no instrumentar la vía aérea y no alterar el control respiratorio, pero como contraparte puede provocar debilidad muscular respiratoria y atenuar la capacidad de toser.

En relación con su impacto respiratorio, se debe revisar el efecto que tiene el bloqueo simpático torácico sobre el tono bronquial y sobre el tono vascular y su consecuencia en la vasoconstricción pulmonar hipóxica.

Existen estudios que reportan episodios de broncoespasmo durante el uso de anestesia regional ${ }^{48,49}$. Pero cuando los estudios son dirigidos a medir el efecto sobre el tono bronquial, se ha visto que el $\mathrm{VEF}_{1}$ cae $(16 \%)$ al usar una alta concentración de anestésicos locales (ropivacaina $0,75 \%)^{50}$ pero que con una concentración menor (ropivacaina $0,25 \%$ ) se produce incluso disminución de la resistencia de la vía aérea y aumento del flujo inspiratorio ${ }^{51}$. El hecho de que en el sistema respiratorio los receptores beta 1 , asociados a las dendritas nerviosas terminales, son mucho menos prevalentes que los beta 2 (1:20), explica porqué el bloqueo simpático regional no provoca aumento del tono bronquial en términos clínicos, lo que podría implicar que la caída del $\mathrm{VEF}_{1}$ se debe al compromiso muscular causado por el uso de anestésico local más concentrado.
El bloqueo simpático puede tener como repercusión la disminución del tono vascular $\mathrm{y}$, de este modo, anular el efecto de la vasoconstricción pulmonar hipóxica, fenómeno fisiológico que disminuye el shunt intrapulmonar. Este efecto ha sido estudiado durante la ventilación monopulmonar (VMP), contexto en el cual este reflejo activo es muy importante. En general se puede afirmar que la anestesia peridural torácica no provoca un compromiso clínicamente importante de la oxigenación durante la VMP. En un estudio con 34 pacientes bajo anestesia general inhalatoria ${ }^{52}, \mathrm{la}_{\mathrm{PaO}}$ y el Qs/ Qt fueron similares cuando se agregó remifentanil endovenoso o bupivacaina $0,5 \%$ vía epidural torácica. Otro estudio ${ }^{53}$ utilizó anestesia intravenosa (fentanilo y propofol) encontrando que la adición de meperidina peridural baja muy levemente la $\mathrm{PaO}_{2}$ y aumenta levemente el Qs/Qt. Por último, la peridural con bupivacaina $0,1 \%$ más morfina, produce una leve caída de la $\mathrm{PaO}_{2}$ y un leve aumento del Qs/Qt cuando fue agregada a anestesia inhalatoria o a TIVA con propofol y fentanilo ${ }^{54}$. Este efecto sobre la $\mathrm{PaO}_{2}$ y la $\mathrm{Qs} / \mathrm{Qt}$ disminuye en la medida que la concentración de anestésico local utilizada es más baja ${ }^{55}$.

Con la evidencia acumulada, ya no se discute que la analgesia postoperatoria alcanzada con la técnica regional es mucho más efectiva que con la analgesia endovenosa ${ }^{56,57}$.

La anestesia/analgesia regional ha sido propuesta como una medida efectiva para disminuir la morbilidad perioperatoria. Sin embargo y, a pesar de numerosos estudios, no existe un consenso para afirmar tal efecto en la población general sometida a todo tipo de cirugías ${ }^{58-62}$. Sin embargo, cuando se analizan subgrupos de pacientes sometidos a un riesgo respiratorio más elevado, como la cirugía cardiaca $^{63}$, el traumatismo torácico ${ }^{64}$ o la cirugía de aorta abdominal ${ }^{65}$, se encuentran datos que hacen pensar que la analgesia regional es capaz de entregar protección en términos respiratorios. Una reciente revisión sistemática también afirma que la anestesia regional disminuye la incidencia de neumonía $^{66}$.

\section{Ventilación protectora}

En los últimos años ha adquirido importancia el Distress Respiratorio del Adulto (ADRS). En su etiopatogenia se ha identificado a la ventilación mecánica, acuñándose el concepto de ventilación protectora a partir del ADRS Network ${ }^{67}$. Este estudio realizado en pacientes con ADRS, demostró que al ocupar un volumen corriente $(\mathrm{Vt})$ menor $(6$ 
versus $12 \mathrm{ml} \cdot \mathrm{kg}^{-1)}$ y una presión meseta menor $(25$ versus $33 \mathrm{cmH}_{2} \mathrm{O}$ ) se logra una disminución de la mortalidad ( $31 \%$ versus $39,8 \%)$. Este concepto se ha extrapolado al ambiente intraoperatorio promoviéndose el uso combinado de volumen corriente bajo, presión de vía aérea baja, PEEP y reclutamiento alveolar.

El daño pulmonar agudo, redefinido en el Consenso de Berlín ${ }^{68}$ como distress respiratorio leve, y la ventilación protectora merecen por sí sola una revisión, lo que excede la intención de esta publicación, pero se mencionarán algunos puntos.

Esta conducta protectora no amerita discusión en aquellos pacientes que van a pabellón y ya tienen establecido un ADRS; así como tampoco en aquellos pacientes que permanecerán en ventilación mecánica por un tiempo prolongado ${ }^{69,70}$. La aplicación de esta conducta "protectora" a todos los pacientes que van a pabellón se ha ido imponiendo por extrapolación más que por evidencia científi$\mathrm{ca}^{71}$. Y la lógica que se ha ido imponiendo es que 1) el no usar ventilación protectora puede hacer daño al paciente o puede dejar de beneficiarlo y, por otro lado, que 2) no se hace daño si se protege innecesariamente.

$\mathrm{Al}$ igual que para la morbilidad cardiovascular, los estudios sobre distress respiratorio perioperatorio se han dirigido a poblaciones de alto riesgo: cirugía cardiotorácica y abdominal. En cirugía torácica se mencionan principalmente los estudios de Licker $^{72}$ y Yang ${ }^{73}$; en especial el primero, entrega cierta evidencia de la utilidad de aplicar una técnica protectora, pero se trata de un estudio retrospectivo con una cohorte de control histórica. De todas formas, la conducta actual en ventilación mono pulmonar es usar Vt entre 5 y $7 \mathrm{ml} \cdot \mathrm{kg}^{-1}$ y una presión meseta que no exceda los $25 \mathrm{cmH}_{2} \mathrm{O}$. El rol del PEEP en este contexto es más discutible.

Para la cirugía abdominal se plantea como principal evidencia el trabajo de Futier de $2013^{74}$, que muestra un gran beneficio en el outcome primario combinado (neumonía, necesidad de ventilación postoperatoria, sepsis, shock séptico, muerte). Sin embargo, en el grupo sin ventilación "protectora" hubo una incidencia de $8 \%$, de neumonía lo que equivale a más del doble de lo publicado ${ }^{5,7}$. Por otra parte, no muestra diferencia en la necesidad de ventilación invasiva (sí de ventilación no invasiva), tampoco en la incidencia de distress (son sólo 200 pacientes por grupo), muestra disminución en la estadía hospitalaria pero no en días de UTI, y en el grupo sin ventilación "protectora" tuvieron una incidencia de $17 \%$ de atelectasias, que es más de 7 veces la publicada ${ }^{7,8}$. Las curvas de KaplanMeier que publica en el Appendix ${ }^{73}$ muestran que la ventilación mecánica y la neumonía comienzan a mostrar diferencias recién a contar del tercer día postoperatorio. Todos estos aspectos impiden que sus resultados se apliquen sin reserva, especialmente en lo referente a la altísima incidencia de atelectasias.

Cabe recordar el carácter multifactorial del daño pulmonar o distress respiratorio, donde la técnica ventilatoria es un factor más. Aún más, los estudios no han discriminado el aporte por separado de los distintos factores de la ventilación, mencionándose reiteradamente un volumen corriente bajo y, sobre todo, una presión meseta baja ${ }^{75,76}$ como las medidas de proteción del pulmón más importantes. El rol del PEEP no ha sido establecido fehacientemente ${ }^{77}$.

Por último, es discutible usar esta técnica protectora en todos los pacientes con el argumento de que con ella no se hace daño. En nuestra práctica clínica hemos visto cómo la aplicación indiscriminada de alguna conducta finalmente puede terminar siendo deletérea para algunos pacientes. Como ejemplo están los almidones de alto peso molecular, la aprotinina, dosis elevadas de heparinas de bajo peso molecular y el uso rutinario de betabloqueadores. Y en esta línea de pensamiento me siento representado por un artículo de anestesistas canadienses publicado en julio de este año ${ }^{78}$ en el que se muestra con elegancia que los efectos negativos de una conducta beneficiosa en un grupo de alto riesgo carecen de importancia, pero que se hacen preponderantes e inaceptables en una población general o de bajo riesgo que no se beneficia de dicha conducta, por lo que llaman a la cautela en la masificación de la conducta "protectora".

Un estudio publicado en septiembre ${ }^{79}$ midió biomarcadores de injuria pulmonar en pacientes con pulmón sano a los 60 minutos de ventilación mecánica, comparando $\mathrm{Vt}$ de $6 \mathrm{ml} \cdot \mathrm{kg}^{-1}$ versus 10 $\mathrm{ml} \cdot \mathrm{kg}^{-1}$, sin encontrar diferencias.

De este modo, los datos actuales, no entregan evidencia contundente o suficiente sobre una indicación perentoria de la ventilación protectora en pacientes sin daño pulmonar sometidos a cirugía ${ }^{80}$.

Un paso intermedio lógico en la investigación sería evaluar la estrategia ventilatoria protectora en poblaciones quirúrgicas con alto riesgo de desarrollar distress respiratorio, antes de hacerlo en población general. En este sentido, un estudio de cohorte multicéntrico aparecido en mayo de este año nos entrega un score que identifica una serie de factores (sepsis, cirugía cardiaca de alto riesgo, cirugía vascular de alto riesgo, cirugía de urgencia, cirrosis, admisión desde un lugar distinto de su hogar, frecuencia respiratoria alta, saturación de $\mathrm{O}_{2}<$ 
$95 \%$, requerimiento de $\mathrm{FiO}_{2}>35 \%$ ) que debería ser útil para este propósito ${ }^{81}$.

\section{CONCLUSIÓN}

La morbilidad respiratoria es un tema preponderante de la medicina perioperatoria, con una gran repercusión clínica. Hay factores asociados al paciente, a la cirugía y a la técnica anestésica.
$\mathrm{La}$ analgesia regional es una herramienta a considerar en grupos de alto riesgo, así como el entrenamiento muscular en pacientes con enfermedad pulmonar crónica. La ventilación protectora tiene un lugar claro en pacientes con distress establecido y en aquellos que serán ventilados mecánicamente en forma prolongada; su aplicación a pacientes con pulmón sano sometidos a cirugía es un tema que se debe definir con mayor investigación clínica.

\section{REFERENCIAS}

1. Fisher BW, Majumdar SR, McAlister FA. Predicting pulmonary complications after nonthoracic surgery: a systematic review of blinded studies. Am J Med 2002; 112: 219-225.

2. Lawrence VA, Dhanda R, Hilsenbeck SG, Page CP. Risk of pulmonary complications after elective abdominal surgery. Chest 1996; 110; 744-750.

3. Warner DO. Preventing Postoperative Pulmonary Complications. The role of the Anesthesiologist. Anesthesiology 2000; 92: 14671472 .

4. Smetana GW. Preoperative pulmonary evaluation. N Engl J Med 1999 Mar 25; 340: 937-944.

5. Arozullah AM, Khuri SF, Henderson WC, Daley J, for the Participants in the National Veterans Affairs Surgical Quality Improvement Program. Ann Inter Med 2001; 135: 847-857.

6. Arozullah AM, Daley J, Henderson WG, et al. Multifactorial Risk Index for Predicting Postoperative Respiratory failure in men After Major Noncardiac Surgery. Annals of Surgery 2000; 232: 242-253.

7. McAlister FA, Khan NA, Straus SE, et al. Accuracy of the Preoperative Assessment in Predicting Pulmoinary Risk after Nonthoracic Surgery. Am J Resp C C Med 2003; 167: 741-744.

8. Canet J, Gallart L1, Gomar C, et al. Prediction of Postoperative Pulmonary Complications in a Population-based Surgical Cohort. Anesthesiology 2010; 113: 1338 -1350 .

9. Pedersen T, Viby-Mogensen J, Ringsted. Anaesthetic practice and postoperative pulmonary complications. Acta Anaesthesiol Scand 1992; 36: 812-818.

10. Dulu A, Pastores SM, Park B, et al. Prevalence and morbidity of acute lung injury and ARDS after lung resection. Chest 2006; 130: 73-78.

11. Jayr C, Matthay MA, Goldstone $\mathrm{J}$, et al. Preoperative and intraoperative factors associated with prolonged mechanical ventilation. A study in patients following major abdominal vascular surgery. Chest 1993; 103: 1231-1236.

12. Berg H, Roed J, Viby-Mogensen J, Mortensen, et al. Residual neuromuscular block is a risk factor for postoperative pulmonary complications. A prospective, randomised, and blinded study of postoperative pulmonary complications after atracurium, vecuronium and pancuronium. Acta Anaesthesiol Scand 1997; 41: 1095-1103.

13. Bluman LG. Mosca L, Newman N, et al. Preoperative smoking habits and postoperative pulmonary complications. Chest 1998; 113: 883889.

14. Tait A, Malviya Sh. Anesthesia for the Child with an Upper Respiratory Tract Infection: Still a Dilemma? Anesth Analg 1998; 86: 706-711.

15. Tusman G, Boöhm SH, Warner $\mathrm{DO}$, et al. Atelectasis and perioperative pulmonary complications in high-risk patients. Current Opinion in Anesthesiology 2012; 25: 1-10.

16. Wightman JA. A prospective survey of the incidence of postoperative pulmonary complications. $\mathrm{Br} \mathrm{J}$ Surg 1968; 55: 85-91.

17. Siafakas NM, Vermeire $P$, Pride $\mathrm{NB}$, et al. Optimal assessment and management of chronic obstructive pulmonary disease (COPD). The European Respiratory Society Task Force. Eur Respir J 1995; 8: 13981420.

18. Warner DO, Warner MA, Offord $\mathrm{KP}$, et al. Airway obstruction and perioperative complications in smokers undergoing abdominal surgery. Anesthesiology 1999; 90 : 372-379.

19. Mannino DM. COPD: epidemio- logy, prevalence, morbidity and mortality, and disease heterogeneity. Chest 2002; 121(5 Suppl): 121S-126S.

20. Pauwels RA, Buist AS, Calverley PM, et al. GOLD Scientific Committee. Global strategy for the diagnosis, management, and prevention of chronic obstructive pulmonary disease. NHLBI/WHO Global Initiative for Chronic Obstructive Lung Disease (GOLD) Workshop summary. Am J Respir Crit Care Med 2001; 163: 1256-1276.

21. Forrest JB, Rehder K, Cahalan MK, et al. Multicenter study of general anesthesia. III. Predictors of severe perioperative adverse outcomes. Anesthesiology 1992; 76: 3-15.

22. Wong DH, Weber EC, Schell MJ, et al. Factors associated with postoperative pulmonary complications in patients with severe chronic obstructive pulmonary disease. Anesth Analg 1995; 80: 276-284.

23. Nomori H, Kobayashi R, Fuyuno $\mathrm{G}$, et al. Preoperative respiratory muscle training. Assessment in thoracic surgery patients with special reference to postoperative pulmonary complications. Chest 1994; 105: 1782-1788.

24. Meyer PA, Mannino DM, Redd SC, et al. Characteristics of adults dying with COPD. Chest. 2002; 122: 2003-2008.

25. Pearce AC, Jones RM. Smoking and anesthesia: preoperative abstinence and perioperative morbidity. Anesthesiology 1984; 61: 576-584.

26. Warner MA, Divertie MB, Tinker JH. Preoperative cessation of smoking and pulmonary complications in coronary artery bypass patients. Anesthesiology 1984; 60: 380-383.

27. Warner MA, Offord KP, Warner $\mathrm{ME}$, et al. Role of preoperative cessation of smoking and other factors in postoperative pulmonary complications: a blinded prospective study of coronary artery bypass patients. Mayo Clin Proc 1989; 64: 609-616. 
28. Vaughan RW, Bauer S, Wise L. Effect of position (semirecumbent versus supine) on postoperative oxygenation in markedly obese subjects. Anesth Analg 1976; 55: $37-41$.

29. Loadsman JA, Hillman DR. Anaesthesia and sleep apnoea. Br J Anaesth 2001; 86: 254-266.

30. El-Solh A, Sikka P, Bozkanat R, et cols. Morbid Obesity in the Medical ICU. Chest 2001; 120; 19891997.

31. Eames WO, Rooke GA, Wu RS, et al. Comparison of the effects of etomidate, propofol, and thiopental on respiratory resistance after tracheal intubation. Anesthesiology 1996; 84: 1307-1311.

32. Nishiyama T, Hanaoka K. Propofol-induced bronchoconstriction: two case reports. Anesth Analg 2001; 93: 645-646.

33. Hepner DL. Propofol-induced bronchoconstriction: asthma or allergy? Anesth Analg 2002 Apr; 94: 1045.

34. Pizov R, Brown RH, Weiss YS, et al. Wheezing during induction of general anesthesia in patients with and without asthma. A randomized, blinded trial. Anesthesiology 1995; 82: 1111-1116.

35. Henzler D, Rossaint R, Kuhlen R. Anaesthetic considerations in patients with chronic pulmonary disease. Current Opinion in Anaesthesiology 2003; 16: 323330.

36. Aye T, Milne B. Ketamine anesthesia for pericardial window in a patient with pericardial tamponade and severe COPD. Can J Anaesth 2002; 49: 283-286.

37. Groeben H, Silvanus MT, Beste $\mathrm{M}$, et al. Combined intravenous lidocaine and inhaled salbutamol protect against bronchial hyperreactivity more effectively than lidocaine or salbutamol alone. Anesthesiology 1998; 89: 862-868.

38. Kim ES, Bishop MJ. Endotracheal intubation, but not laryngeal mask airway insertion, produces reversible bronchoconstriction. Anesthesiology 1999; 90: 391-394.

39. Natalini G, Franceschetti ME, Pletti C, et al. Impact of laryngeal mask airway and tracheal tube on pulmonary function during the early postoperative period. Acta Anaesthesiol Scand 2002; 46: 525.

40. Basta SJ, Savarese JJ, Ali HH, et al. Histamine-releasing potencies of atracurium besylate (BW 33A), metocurine and d-tubocurarine. Anesthesiology 1982; 57: A261.
41. Basta SJ, Savarese JJ, Ali HH, et al. Histamine-releasing potencies of atracurium, dimethyl tubocurarine and tubocurarine. Br J Anaesth 1983; 55 (Suppl): 105S-106S.

42. Savarese JJ, Ali HH, Basta SJ, et al. The clinical neuromuscular pharmacology of mivacurium chloride (BW B1090U). Anesthesiology 1988; 68: 723-732.

43. Rooke GA, Choi JH, Bishop MJ. The effect of isoflurane, halothane, sevoflurane, and thiopental/ nitrous oxide on respiratory system resistance after tracheal intubation. Anesthesiology 1997; 86: 12941299.

44. Volta CA, Alvisi V, Petrini S, et al. The effect of volatile anesthetics on respiratory system resistance in patients with chronic obstructive pulmonary disease. Anesth Analg 2005; 100: 348-353.

45. Habre W, Petak F, Sly PD, et al. Protective effects of volatile agents against methacholine-induced bronchocoriction in rats. Anesthesiology 2001; 94: 348.

46. Dikmen Y, Eminoglou E, Salihoglou Z, et al. Pulmonary mechanics during isoflurane, sevoflurane and desflurane anesthesia. Anaesthesia 2003; 58: 745.

47. Nyktari VG, Papaioannou AA, Prinianakis G, et al. Effect of the physical properties of isoflurane, sevoflurane and desflurane on pulmonary resistance in a laboratory lung model. Anesthesiology 2006; 104: 1202.

48. Shnider SM, Papper EM. Anesthesia for the asthmatic patient. Anesthesiology 1961; 22: 886-892.

49. Warner DO, Warner MA, Barnes $\mathrm{RD}$, et al. Perioperative respiratory complications in patients with asthma. Anesthesiology 1996; 85: 460-467.

50. Groeben H, Schwalen A, Irsfeld S, et al. Pulmonary sympathetic denervation does not increase airway resistance in patients with chronic obstructive pulmonary disease (COPD). Acta Anaesthesiol Scand 1995; 39: 523-526.

51. Gruber EM, Tschernko EM, Kritzinger M, et al. The Effects of Thoracic Epidural Analgesia with Bupivacaine $0.25 \%$ on Ventilatory Mechanics in Patients with Severe Chronic Obstructive Pulmonary Disease. Anesth Analg 2001; 92 : 1015-1019.

52. Chow MY, Goh MH, Boev SK, et al. The Effects of Remifentanil and Thoracic Epidural on Oxygenation and Pulmonary Shunt Fraction
During One-Lung Ventilation. J Cardiothorac Vasc Anesth 2003; 17: 69-72.

53. Garutti I, Cruz P, Olmedilla L, et al. Effects of Thoracic Epidural Meperidine on Arterial Oxygenation During One-Lung Ventilation in Thoracic Surgery. J Cardiothorac Vasc Anesth 2003; 17: 302-305.

54. Özcan PE, Sentürk M, Zungur Ulke Z, et al. Effects of thoracic epidural anaesthesia on pulmonary venous admixture and oxygenation during one-lung ventilation. Acta Anaesth Scand 2007; 51: 11171122.

55. Xu Y, Tan Z, Wang S, et al. Effect of Thoracic Epidural Anesthesia with Different Concentrations of Ropivacaine on arterial oxygenation during one-lung Ventilation. Anesthesiology 2010; 112: 11461154.

56. Block BM, Liu SS, Rowlingson AJ, et al. Efficacy of postoperative epidural analgesia: a meta-analysis. JAMA 2003; 290: 2455-2463.

57. Wu CL, Cohen SR, Richman JM, et al. Efficacy of Postoperative Patient-controlled and Continuous Infusion Epidural Analgesia versus Intravenous Patient-controlled Analgesia with Opioids. A Metaanalysis. Anesthesiology 2005; 103: 1079-1088.

58. Ballantyne JC. The comparative effects of postoperative analgesic therapies on pulmonary outcome: cumulative meta-analysis or randomized, controlled trials. Anesth Analg 1998; 86: 598-612.

59. Rodgers A. Reduction of post operative mortality and mortality with epidural or spinal anaesthesia: results from overview of randomized trials. Br Med J 2000; 321: 1493-1497.

60. Norris EJ. Double-masked randomized trial comparing alternate combinations of intraoperative anestesia and postoperative analgesia in abdominal aortic surgery. Anesthesiology 2001; 95: 10541067.

61. Park WY. Effect of epidural anesthesia and perioperative outcome: a randomized, controlled veterans affairs cooperative study. Ann Surg 2001; 234: 560-569.

62. Rigg JR. Epidural anaesthesia and analgesia and outcome of major surgery: a randomized trial. Lancet 2002; 359: 1276-1282.

63. Liu SS, Block BM, Wu CL. Effects of perioperative central neuraxial analgesia on outcome after coronary artery bypass surgery: a meta- 
analysis. Anesthesiology 2004; 101: 153-161.

64. Bulger EM, Edwards TH, Klotz P, et al. Epidural analgesia improves outcome after multiple rib fractures. Surgery 2004; 136: 426-430.

65. Nishimori M, Low JHS, Zheng H, et al. Epidural pain relief versus systemic opioid-based pain relief for abdominal aortic surgery. Cochrane Database of Systematic Reviews 2012.

66. Guay J, Choi P, Suresh S, et al. Neuroaxial blockade for the prevention of postoperative mortality and major morbidity: an overview of Cochrane systematic reviews. Cochrane Database of Systematic Reviews 2014, Issue 1. Art. No.: CD010108. DOI: 10.1002/14651858.CD010108. pub2.

67. de Campos T. Ventilation with lower tidal volumes as compared with traditional tidal volumes for acute lung injury and the acute respiratory distress síndrome. ADRS Network. N Engl J Med 2000; 342: 1301-1308.

68. Ranieri VM, Rubenfeld GD, Thompson BT, et al. Acute respiratory \& distress syndrome: the Berlin definition. JAMA 2012; 307: 2526-2533.

69. Determann RM, Royakkers A, Wolthuis EK, et al. Ventilation with lower tidal volumes as compared with conventional tidal volumes for patients without acute lung injury: a preventive randomized controlled trial. Critical Care 2010, 14:R1 http://ccforum.com/content/14/1/ $\mathrm{R} 1$.

70. Xiaoming J, Malhotra A, Saeed M, et al. Risk Factors for Acute Respiratory Distress Syndrome in Patients Mechanically Ventilated for Greater Than 48 Hours. Chest 2008; 133: 853-861.

71. Schultz MJ, Haitsma JJ, Slutsky AS, et al. What Tidal Volumes Should Be Used in Patients without Acute Lung Injury? Anesthesiology 2007; 106: 1226-1231.

72. Licker M, Diaper J, Villiger Y, et al. Impact of intraoperative lung-protective interventions in patients undergoing lung cancer surgery. Critical Care 2009, 13:R41 (doi:10.1186/cc7762).

73. Yang M, Ahn HJ, Kim K, et al. Does a Protective Ventilation Strategy Reduce the Risk of Pulmonary Complications After Lung Cancer Surgery? A Randomized Controlled Trial. CHEST 2011; 139: 530-537.

74. Futier E, Constantin JM, PaugamBurtz C, et al. A Trial of Intraoperative Low-Tidal-Volume Ventilation in Abdominal Surgery. N Engl J Med 2013; 369: 428-437.

75. Petrucci N, de Feo C. Lung protective ventilation strategy for the acute respiratory distress syndrome. Cochrane Database of
Systematic Reviews 2013, Issue 2. Art. No.: CD003844. DOI: 10.1002/14651858.CD003844. pub4.

76. Prescott HC, Brower RG, Cooke $\mathrm{CR}$, et al. Factors Associated With Elevated Plateau Pressure in Patients With Acute Lung Injury Receiving Lower Tidal Volume Ventilation. Crit Care Med 2013; 41: 756-764.

77. Luengo A, Carvajal C. Atelectasias intraoperatorias, mecanismos de formación y estrategias de prevención. Rev Chil Anest 2013; 42: 167-179.

78. Goldenberg NM, Steinberg BE, Lee WL, et al. Lung-protective Ventilation in the Operating Room. Time to Implement? Anesthesiology 2014; 121: 184-188.

79. Fernández-Bustamante A, Klawitter J, Repine JE, et al. Effect of Tidal Volume on Lung Injury Biomarkers in Surgical Patients with Healthy Lungs. Anesthesiology 2014; 121 : 469-481.

80. Wrigge H, Pelosi P. Tidal Volume in Patients with Normal Lungs during General Anesthesia. Lower the Better? Anesthesiology 2011; 114: 1102-1110.

81. Kor DJ, Lingineni RK, Gajic O. Predicting Risk of Postoperative Lung Injury in Highrisk Surgival Patients. A Multicenter Cohort Study. Anesthesiology 2014; 120: 1168-1181.

\section{Correspondencia a: \\ Dr. Mauricio Ramos Garrido \\ mauroramosg@gmail.com}

